\title{
Nothing up his sleeve: Decompensation after bariatric surgery
}

Charisse Colvin, MD, Winnie Tsai, DO, Andrew L. Silverman, MD, Jenna Taglienti, MD, and Binu Chacko, MD

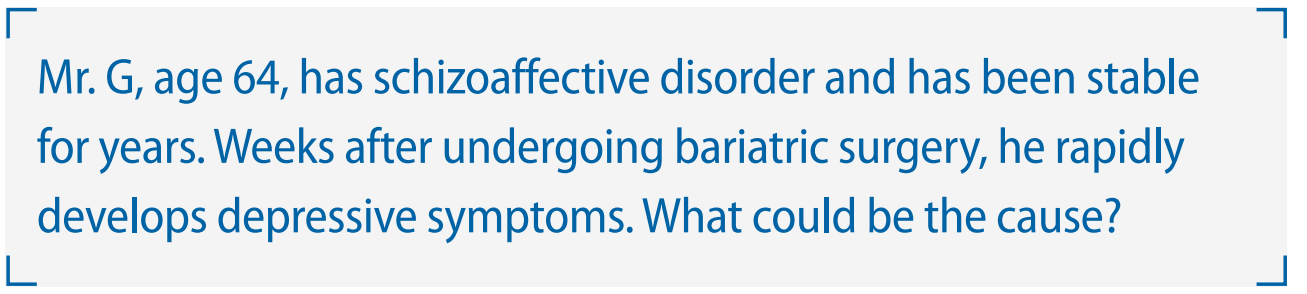

\section{CASE Sudden-onset low mood}

Mr. G, age 64, is obese (body mass index [BMI] $37 \mathrm{~kg} / \mathrm{m}^{2}$ ) and has a history of schizoaffective disorder. He is recovering from a sleeve gastrectomy, a surgical weight-loss procedure in which a large portion of the stomach is removed. Seven weeks after his surgery, he experiences a sudden onset of "low mood" and fears that he will become suicidal; he has a history of suicide attempts. Mr. G calls his long-term outpatient clinic and is advised to go to the emergency department (ED).

For years, Mr. G had been stable in a group home setting, and had always been adherent to treatment and forthcoming about his medications with both his bariatric surgeon and psychiatrist. Within the last month, he had been seen at the clinic, had no psychiatric symptoms, and was recovering well from the sleeve gastrectomy.

\section{HISTORY A stable regimen}

Mr. G's psychiatric symptoms initially developed when he was in his 20s, during a time in which he reported using "a lot of drugs." He had multiple suicide attempts, and multiple inpatient and outpatient treatments. He was diagnosed with schizoaffective disorder.

Mr. $G$ has been stable on medications for the last 2 years. His outpatient psychotropic regimen is divalproex sodium extended- release (ER), 2,500 mg every night at bedtime; iloperidone, $8 \mathrm{mg}$ twice a day; escitalopram, $10 \mathrm{mg} / \mathrm{d}$; and mirtazapine, $30 \mathrm{mg}$ every night at bedtime.

In the group home, Mr. G spends his days socializing, studying philosophy, and writing essays. He hopes to find a job in the craftsman industry.

Mr. G's medical history includes obesity (BMl: $37 \mathrm{~kg} / \mathrm{m}^{2}$ ). Since the surgery, he has been receiving omeprazole, $40 \mathrm{mg} / \mathrm{d}$, a proton pump inhibitor (PPI), to decrease the amount of acid in his stomach. Three weeks after surgery, he had an unremarkable postoperative outpatient psychiatry visit. Divalproex sodium ER was maintained at the pre-surgical dose of $2,500 \mathrm{mg} / \mathrm{d}$.

continued

Dr. Colvin is a PGY-4 Psychiatry Resident, Department of Psychiatry, Donald and Barbara Zucker School of Medicine at Hofstra/Northwell, Mather Hospital Northwell Health, Port Jefferson, New York. Dr. Tsai is a PGY-1 Psychiatry Resident, Department of Psychiatry, Donald and Barbara Zucker School of Medicine at Hofstra/Northwell, Mather Hospital Northwell Health, Port Jefferson, New York. Dr. Silverman is a PGY-1 Psychiatry Resident, Department of Psychiatry, Donald and Barbara Zucker School of Medicine at Hofstra/Northwell, Mather Hospital Northwell Health, Port Jefferson, New York. Dr. Taglienti is Program Director, Department of Psychiatry, Donald and Barbara Zucker School of Medicine at Hofstra/Northwell, Mather Hospital Northwell Health, Port Jefferson, New York. Dr. Chacko is Assistant Program Director, Department of Psychiatry, Donald and Barbara Zucker School of Medicine at Hofstra/Northwell, Mather Hospital Northwell Health, Port Jefferson, New York.

\section{Disclosures}

The authors report no financial relationships with any companies whose products are mentioned in this article, or with manufacturers of competing products.

doi: $10.12788 /$ cp. 0100

\section{How would you} handle this case?

Answer the challenge questions at MDedge.com/ psychiatry and see how your colleagues responded 


\section{Clinical Point}

The treatment team believed Mr. G's decompensation had to be related to a recent event, such as his surgery and/or medication change

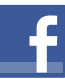

Discuss this article at www.facebook.com/ MDedgePsychiatry

\section{EVALUATION Depressed and} frightened

In the ED, Mr. G's vitals are normal, but his serum valproic acid (VPA) level is $33.68 \mu \mathrm{g} /$ $\mathrm{mL}$ (therapeutic range: 50 to $125 \mu \mathrm{g} / \mathrm{mL}$ ), despite being compliant with treatment. Mr. $G$ is discharged from the ED and told to follow up with his outpatient psychiatrist the next day.

At his outpatient psychiatry appointment, Mr. G's vital signs are normal, but he reports increasing depression and worsened mood. On mental status examination, Mr. G's appearance is well groomed, and no agitation nor fidgeting are observed. His behavior is cooperative but somewhat disorganized. $\mathrm{He}$ is perseverative on "feeling so low." He has poor eye contact, which is unusual for him. Mr. G's speech is loud compared with his baseline. Affect is congruent to mood, which he describes as "depressed and frightened." $\mathrm{He}$ is also noted to be irritable. His thought process is abstract and tangential, which is within his baseline. Mr. G's thought content is fearful and negativistic, despite his usual positivity and optimism. He denies hallucinations and is oriented to time, place, and person. His judgment, attention, and memory are all within normal limits.

\section{Why might Mr. G's serum VPA level be low?}

a) his claim of being compliant with medication is not true

b) the bariatric surgery impaired absorption of the medication

c) the bloodwork was done at the wrong time of the day, leading to a false reading of the serum VPA level

d) a laboratory error occurred when measuring the VPA concentration

e) another medication is preventing the release of divalproex sodium ER

\section{The authors' observations}

The psychiatrist rules out malingering/ nonadherence due to Mr. G's long history of treatment compliance, as evidenced by his past symptom control and therapeutic serum VPA levels. Mr. G was compliant with his postoperative appointments and has been healing well. Therefore, the treatment team believed that Mr. G's intense and acute decompensation had to be related to a recent change. The notable changes in $\mathrm{Mr}$. G's case included his sleeve gastrectomy, and the addition of omeprazole to his medication regimen.

The treatment team observed that Mr. G had a long history of compliance with his medications and his symptoms were consistent with a low serum VPA level, which led to the conclusion that the low serum VPA level measured while he was in the ED was likely accurate. This prompted the team to consider Mr. G's recent surgery. It is well documented that some bariatric surgeries can cause poor absorption of certain vitamins, minerals, and medications. However, Mr. G had a sleeve gastrectomy, which preserves absorption. At this point, the team considered if the patient's recent medication change was the source of his low VPA level.

The psychiatrist concluded that the issue must have been with the addition of omeprazole because Mr. G's sleeve gastrectomy was noneventful, he was healing well and being closely monitored by his bariatric surgeon, and this type of surgery preserves absorption. Fortunately, Mr. G was a good historian and had informed his psychiatrist about the addition of omeprazole after his sleeve gastrectomy. The psychiatrist knew acidity was important for the absorption of some medications. Although she was unsure as to whether the problem was a lack of absorption or lack of delivery, the psychiatrist knew a medication change was necessary to raise Mr. G's serum VPA levels.

\section{TREATMENT A change in divalproex formulation}

The psychiatrist switches Mr. G's formulation of divalproex sodium ER, 2,500 mg/d, 
to valproic acid immediate-release (IR) liquid capsules. He receives a total daily dose of $2,500 \mathrm{mg}$, but the dosage is split into 3 times a day. The omeprazole is continued to maintain the postoperative healing process, and he receives his other medications as well (iloperidone, $8 \mathrm{mg}$ twice a day; escitalopram, $10 \mathrm{mg} / \mathrm{d}$; and mirtazapine, $30 \mathrm{mg}$ every night at bedtime).

\section{Why would a change in valproate formulation lead to increased serum VPA levels for Mr. G? \\ a) the IR formulation is always absorbed better than the ER formulation \\ b) the release of the medication in IR formu- lation is not affected by gastric acid, and therefore is not affected by the surgery nor the prescribed omeprazole \\ c) this is an anomaly and therefore an unusual case report}

\section{The authors' observations}

The key component to creating a treatment plan for Mr. G centered on understanding drug metabolism and delivery. Acidity plays a role in dissolution of many medications, which led the team to surmise that the PPI, omeprazole, was the culprit. Through research, they understood that the divalproex sodium ER formulation needed a more acidic environment to dissolve, and therefore, an IR formulation was needed.

\section{Different formulations, different characteristics}

Medications can be produced in different formulations such as IR, delayed-release (DR), and ER formulations. Different formulations may contain the same medication at identical strengths; however, they may not be bioequivalent and should be titrated based on both the properties of the medication and the release type. ${ }^{1}$

Immediate-release formulations are developed to dissolve without delaying or prolonging absorption of the medication. These formulations typically include "superdisintegrants" containing croscarmellose sodium ${ }^{2}$ so that they disintegrate, de-aggregate, and/or dissolve when they come into contact with water or the gastrointestinal tract. ${ }^{3-7}$

Delayed-release formulations rely on the gastrointestinal $\mathrm{pH}$ to release the medication after a certain amount of time has elapsed due to the enteric coating surrounding the tablet. This enteric coating prevents gastric mucosa/gastric juices from inactivating an acid-labile medication. ${ }^{8}$

Extended-release formulations, such as the divalproex sodium ER that was originally prescribed to Mr. G, are designed to release the medication in a controlled manner over an extended period of time, and at a predetermined rate and location following administration..$^{8-9}$ The advantage of this type of formulation is that it can be used to reduce dose frequency and improve adherence. ${ }^{10}$ Extended-release formulations are designed to minimize fluctuations in serum drug concentration between doses, ${ }^{11}$ thereby reducing the risk of adverse effects. ${ }^{12,13} \mathrm{~A}$ list of some common extended-release psychiatric medications is shown in the Table (page 18).

The 5 oral formulations of medications that contain valproic acid include:

- syrup

- capsule

- sprinkle

- enteric-coated delayed-release and extended-release

A parenteral form via IV is available for patients who are unable to swallow.

\section{Absorption vs delivery}

Any gastric bypass surgery can have postoperative complications, one of which can include absorption deficiencies of vitamins and minerals. Sleeve gastrectomy has the least amount of absorption-related nutritional deficiencies. ${ }^{14}$ Additionally, this procedure preserves the stomach's ability to produce gastric acid. Therefore, regardless of formulation, there should be no

\section{Clinical Point}

Divalproex sodium ER needs a more acidic environment to dissolve, so Mr. G was switched to an IR formulation 


\section{Clinical Point}

Switching Mr. G to an IR formulation bypassed the need for a low $\mathrm{pH}$ and the VPA was delivered and absorbed

\section{Table}

\section{Different psychiatric medication formulations ${ }^{a}$}

\begin{tabular}{l:l} 
Medication & Absorption/delivery \\
\hline Divalproex sodium ER tablet & Hydrophilic matrix \\
\hline Venlafaxine ER capsule & $\begin{array}{l}\text { Drug release is controlled by diffusion through the coating membrane } \\
\text { on the spheroids and is not pH-dependent }\end{array}$ \\
\hline Bupropion SR tablet & Hydrophilic matrix \\
\hline Bupropion XL tablet & A moisture-barrier coating surrounding a control-release coating \\
\hline Paroxetine tablet & A moisture-barrier coating surrounding a hydrophilic matrix \\
\hline Methylphenidate ER tablet & An immediate-release drug coating surrounding a tri-layer core. The \\
& tri-layer core is composed of 2 drug layers containing the drug, and a \\
\hline Methylphenidate ER capsule & Enteric-coated, delayed-release beads (made by applying a coating of \\
& a water-insoluble, dissolution rate-controlling polymer to IR beads) \\
\hline Methylphenidate LA capsule & Contains 50/50 IR/ER beads \\
\hline Clonidine ER & Hydrophilic matrix \\
\hline aAll formulations can vary depending & on the manufacturer \\
\hline ER: extended-release; IR: immediate-release; LA: long-acting; XL: extended-release
\end{tabular}

initial postsurgical need to change psychotropic medication formulations. However, because VPA is related to B-vitamin deficiency, supplementation can be considered.

Omeprazole is a PPI that increases $\mathrm{pH}$ in the stomach and is often prescribed to promote healing of gastric surgery. However, in Mr. G's case, omeprazole created a nonacidic environment in his stomach, which prevented the divalproex sodium ER formulation from being dissolved and the medication from being delivered. Mr. G's absorption ability was preserved, which was confirmed by his rapid recovery and increased serum VPA levels once he was switched to the IR formulation. There is no literature supporting a recommended length of time a patient can receive omeprazole therapy for sleeve gastrectomy; this is at the surgeon's discretion. Mr. G's prescription for omeprazole was for 3 months.

\section{Proper valproate dosing}

In Mr. G's case, it could be hypothesized that the VPA dosing was incorrect. For mood disorders, oral VPA dosing is $25 \mathrm{mg} / \mathrm{kg} / \mathrm{d}$. Mr. G lost 40 pounds, which would translate to a 450-mg reduction in dose. Despite maintaining his original dose, his serum VPA levels decreased by almost $50 \%$ and could not be attributed to trough measurement. In this case, Mr. G was prescribed a higher dose than needed given his weight loss.

Divalproex sodium ER is a hydrophilic matrix tablet that requires a low $\mathrm{pH}$ to dissolve. Switching to an IR formulation bypassed the need for a low $\mathrm{pH}$ and the VPA was delivered and absorbed. Mr. G was always able to absorb the medication, but only when delivered. The Table lists other psychiatric medications that clinicians should be aware of that utilize similar hydrophilic matrix technology to slowly release medications through the gastrointestinal tract and also require low $\mathrm{pH}$ to release the medication from the tablet.

\section{OUTCOME Stable once again}

Two and a half weeks after his medication formulation is changed from divalproex sodium ER to IR, Mr. G shows improvement in his symptoms. His serum VPA level is $52 \mu \mathrm{g} / \mathrm{mL}$, which is within therapeutic limits. He continues 
receiving his previous medications as well. He reports "feeling much better" and denies having any depressive symptoms nor anxiety. Mr. $\mathrm{G}$ is able to maintain eye contact, and has positive thought content, improved organization of thinking, and retained abstraction.

\section{References}

1. Wheless JW, Phelps SJ. A clinician's guide to oral extendedrelease drug delivery systems in epilepsy. J Pediatr Pharmacol Ther. 2018;23(4):277-292.

2. Jaimini M, Ranga S, Kumar A, et al. A review on immediate release drug delivery system by using design of experiment. J Drug Discov Therap. 2013;1(12):21-27.

3. Bhandari N, Kumar A, Choudhary A, et al. A review on immediate release drug delivery system. Int Res J Pharm App Sci. 2014;49(1):78-87.

4. Eatock J, Baker GA. Managing patient adherence and quality of life in epilepsy. Neuropsychiatr Dis Treat. 2007;3(1): 117-131.

5. Manjunath R, Davis KL, Candrilli SD, et al. Association of antiepileptic drug nonadherence with risk of seizures in adults with epilepsy. Epilepsy Behav. 2009;14(2): 372-378.

6. Samsonsen C, Reimers A, Bråthen G, et al. Nonadherence to treatment causing acute hospitalizations in people with epilepsy: an observational, prospective study. Epilepsia. 2014;55(11):e125-e128. doi: 10.1111/epi.12801

7. Mangal M, Thakral S, Goswami M, et al. Superdisintegrants: an updated review. Int Pharmacy Pharmaceut Sci Res. 2012;2(2):26-35.

8. Tablets. United States Pharmacopeia. Accessed January 21, 2021. http://www.pharmacopeia.cn/v29240usp29nf24s0 c1151s87.html

9. Holquist C, Fava W. FDA safety page: delayed-vs. extendedrelease Rxs. Drug Topics. Published July 23, 2007. Accessed January 21, 2021. https://www.drugtopics.com/view/fdasafety-page-delayed-release-vs-extended-release-rxs

10. Qiu Y, Zhou D. Understanding design and development of modified release solid oral dosage forms. J Validation Technol. 2011;17(2):23-32.

\section{Related Resources}

- Monte SV, Russo KM, Mustafa E. Impact of sleeve gastrectomy on psychiatric medication use and symptoms. J Obes. 2018; 2018:8532602. doi: 10.1155/2018/8532602

- Qiu Y, Zhou D. Understanding design and development of modified release solid oral dosage forms. J Validation Technol. 2011;17(2):23-32.

- ObesityHelp, Inc. https://www.obesityhelp.com/medicationsafter-bariatric-surgery-wls/

Drug Brand Names

\section{Bupropion - Wellbutrin, Zyban}

Clonidine ER - Kapvay

Divalproex sodium extended-

release tablets - Depakote ER

Escitalopram • Lexapro

lloperidone · Fanapt

Methylphenidate ER

tablet $\cdot$ Concerta

Methylphenidate ER capsule •

Metadate, Jornay

Methylphenidate LA

capsule • Ritalin LA

11. Perucca E. Extended-release formulations of antiepileptic drugs: rationale and comparative value. Epilepsy Curr. 2009;9(6):153-157.

12. Bialer M. Extended-release formulations for the treatment of epilepsy. CNS Drugs. 2007;21(9):765-774.

13. Pellock JM, Smith MC, Cloyd JC, et al. Extended-release formulations: simplifying strategies in the management of antiepileptic drug therapy. Epilepsy Behav. 2004;5(3): 301-307.

14. Sarkhosh K, Birch DW, Sharma A, et al. Complications associated with laparoscopic sleeve gastrectomy for morbid obesity: a surgeon's guide. Can J Surg 2013; 56(5):347-352.

\section{Bottom Line}

All medication changes should be identified at each visit. Many extended-release psychiatric medications require lower $\mathrm{pH}$ to release the medication from the tablet. When evaluating nonresponse to psychotropic medications, anything that affects $\mathrm{pH}$ in the stomach should be considered. 